\title{
Desenvolvimento de Habilidades Metacognitivas: Capacitação de Professores de Ensino Fundamental
}

\author{
Development of Metacognitive Strategies: Qualification \\ of Elementary School Teachers
}

\author{
Fernanda de Bastani Busnello*, Graciela Inchausti de Jou \& Tânia Maria Sperb \\ Universidade Federal do Rio Grande do Sul, Porto Alegre, Brasil
}

\begin{abstract}
Resumo
Pesquisas proporcionaram evidências importantes sobre a relação entre estratégias específicas de estudo e um alto desempenho escolar. O objetivo desta investigação é elaborar e avaliar um curso de capacitação para professores de ensino fundamental, com a finalidade de desenvolver as habilidades cognitivas, metacognitivas e motivacionais para a aprendizagem em seus alunos. Utilizou-se um delineamento pré e pós-teste. Participaram 10 professores de $5^{\text {a }}$ série e 54 alunos. Os instrumentos utilizados junto aos alunos foram o Raven, a Escala de Avaliação para Motivação para Aprender, a Escala de Estratégias de Aprendizagem e o Teste de Desempenho Escolar. Para os professores, foram utilizados o Questionário Metacognitivo e um questionário de autoavaliação. Nas análises intra e entre grupos, os resultados mostraram o efeito tempo para a maioria das variáveis e o efeito grupo apenas para a variável estratégias de aprendizagem. Conclui-se que os professores podem atuar explicitamente como mediadores no desenvolvimento das habilidades cognitivas e metacognitivas de aprendizagem.

Palavras-chave: Cognição, metacognição, ensino-aprendizagem.

Abstract

Researches brought important evidences on the relation between specific strategies of study and elementary school performance. The objective of this investigation is to elaborate and evaluate a qualification course for elementary school teachers, aiming at developing their students learning of cognitive, metacognitive and motivational abilities. A pre-posttest design was used. Ten $5^{\text {th }}$ grade teachers and 54 students participated in the study. The instruments used with the students were the Raven, the Scale for the Evaluation of Motivation to Learn, the Strategies for Learning Scale and the School Performance Test. For the teachers, the Metacognitive Questionnaire and a self-evaluation questionnaire were used. Results of the within and between group analyses showed a time effect for most variables and a group effect only for the strategies of learning variable. It is possible to conclude that teachers may explicitly act as mediators in the development of cognitive abilities of learning.

Keywords: Cognition, metacognition, teaching/learning.
\end{abstract}

A Psicologia Cognitiva, mediante pesquisas experimentais, tem proporcionado importantes evidências sobre a relação entre estratégias específicas de estudo e um alto desempenho escolar (Bieleaczyc, Pirolli, \& Brown, 1995). Com esse objetivo foram desenvolvidas, nas últimas décadas, várias pesquisas que investigaram os fatores envolvidos na aprendizagem eficiente, como a motivação (Neves \& Boruchovitch, 2004), a aprendizagem autorregulada (Kramarski \& Michalsky, 2009;

\footnotetext{
* Fernanda de Bastani Busnello, está atualmente na Unidade Estratégica de Resultados da Educação, Serviço Social da Indústria do Rio Grande do Sul (SESI-RS).

Endereço para correspondência: Rua Fernando Carneiro, 269, Porto Alegre, RS, Brasil, 91330-100. Tel.: (51) 3328 4134. E-mail: grajou@gmail.com, febastani@yahoo.com.br e sperbt@terra.com.br
}

Schraw, Crippen, \& Hartley, 2006; Zimmerman, 2001), a aprendizagem e automonitoramento (Rivers, 2001) e a metacognição e aprendizagem eficiente (Blakey \& Spence, 2000; Hartman, 2002; Sternberg, 2002). Assim, a possibilidade de criar um corpo de conhecimento sobre as habilidades de aprendizagem acadêmica tornou-se um atrativo para a compreensão dos processos de ensino e aprendizagem.

As práticas educacionais fundamentadas na teoria cognitiva preocupam-se em desenvolver as habilidades cognitivas, metacognitivas e motivacionais dos aprendizes no contexto escolar (Resnick, 1981; Schraw et al., 2006). Tais práticas dão ênfase ao como aprender, utilizando o que aprender como meio para desenvolver as habilidades específicas de aprendizagem (Salas \& Cannon-Bowers, 2001). Nessa linha, surgiram pesquisas de intervenção que, com base na psicologia cognitiva, 
visaram o aprimoramento do potencial de aprendizagem através do desenvolvimento de habilidades metacognitivas (Boruchovitch \& Mercuri, 1999; Forest-Pressley \& Waller, 1984; Garner, 1987; Jou, 2001; Jou \& Sperb, 2008, 2009; Klauer, 1994; Kramarski \& Michalsky, 2009; Oliveira et al., 1989; Thiede \& Anderson, 2003; Vidal-Abarca, 1990; Vieira, 1999).

Portanto, elucidar as estratégias apropriadas para a aprendizagem eficiente permitiria elaborar planos pedagógicos com o intuito de ensinar também procedimentos e estratégias de aprendizagem no ambiente formal de sala de aula. Neste contexto, alunos com dificuldades de aprendizagem seriam especialmente beneficiados, a partir do conhecimento e do aprimoramento das estratégias que utilizam para aprender.

O modelo de aprendizagem eficiente de Ertmer e Newby (1996) inclui os processos de planejar, controlar e refletir, destacando o caráter consciente desses processos. Na aprendizagem eficiente, o sujeito está consciente do conhecimento específico, das metas que tem que alcançar, das estratégias necessárias para alcançá-las, assim como do próprio processo no momento mesmo de sua ocorrência. Essa atividade on-line, como muitos autores a caracterizam, utilizando a metáfora computacional, traz como consequência o processo autorregulador (Jou, 2001; Jou \& Sperb, 2006).

Schaefer, Pavan, Amaral e Jou (2006) desenvolveram um estudo com aprendizes eficientes no qual investigaram as características desses alunos e sua relação com o alto desempenho acadêmico. Os resultados mostraram que, além de um alto nível intelectual, os estudantes utilizavam com facilidade as estratégias metacognitivas descritas na literatura e mostraram autonomia na condução de sua aprendizagem.

Segundo Rivers (2001), é possível aprimorar estratégias metacognitivas no processo de aprendizagem, visto que as pessoas podem ser ensinadas a automonitorar e a controlar o seu desempenho, passando da heteronomia à autonomia, ou seja, assumindo o controle do próprio processo de aprendizagem. Zimmerman (2001) também diz que, embora a aprendizagem autorregulada não seja adquirida espontaneamente, ela pode ser desenvolvida através de ambientes que ensinem os estudantes a controlarem sua própria aprendizagem.

Por outro lado, os alunos com dificuldades de aprendizagem, em geral, não conhecem as estratégias de monitoramento da aprendizagem ou não as utilizam, quer seja de forma automática, quer seja de forma consciente (Armbruster \& Brown, 1984). O fracasso de muitos alunos em aprender não pode ser atribuído somente a problemas cognitivos, devendo-se considerar, também, as dificuldades metacognitivas. Muitas vezes, a causa do problema pode estar no fato de que os alunos não conseguem utilizar seus saberes e habilidades e também têm dificuldades em transferi-los para outras situações (Davis, Nunes, \& Nunes, 2005).
A metacognição é definida como a capacidade do ser humano de monitorar e autorregular os processos cognitivos (Flavell, 1987; Nelson \& Narens, 1996; Sternberg, 2002). Segundo o enfoque do Processamento de Informação (PI), para que isso aconteça, o sistema cognitivo do indivíduo estaria provido de um subsistema de controle, o qual monitoraria e regularia os processos cognitivos. Esse controle metacognitivo, na maioria das vezes, e especialmente em crianças pequenas, acontece sem que se tenha consciência dos processos de monitoramento e de autorregulação. Entretanto, à medida que os processos cognitivos são mais exigidos por situações de vida mais complexas, os processos metacognitivos tornam-se mais conscientes. Nessas situações, a metacognição é definida como a capacidade de refletir conscientemente sobre os próprios processos cognitivos e metacognitivos (Jou, 2001; Jou \& Sperb, 2006). Nelson e Narens (1996) focalizam os processos de monitoração e autorregulação da habilidade metacognitiva mediante o estudo dos mecanismos reflexivos envolvidos nas respostas obtidas em situações do cotidiano. Segundo o modelo destes autores, o automonitoramento emerge antes da autorregulação.

Pesquisas recentes na área de neuropsicologia, que estudam as funções executivas, sugerem uma separação entre metacognição e cognição. Assim, a metacognição consiste de dois tipos de comportamento: autoavaliação e autogerência (Rivers, 2001). Os processos de seleção, controle e monitoração são abordados tanto pela psicologia cognitiva (metacognição) quanto pela neuropsicologia (funções executivas), mas, como alerta Bosa (2001), é preciso demarcar os limites entre ambas as áreas para evitar o uso indiscriminado dos termos metacognição e funções executivas.

O termo metacognição não se refere somente ao conhecimento sobre a cognição. Atualmente, entende-se metacognição como uma fase de processamento de alto nível, adquirida e desenvolvida pela experiência e pelo acúmulo do conhecimento específico. Em função desse processamento supraordenado, o indivíduo consegue monitorar, autorregular e elaborar estratégias para potencializar sua cognição (Flavell, 1987). Dessa forma, a metacognição tornou-se de grande importância para as propostas de instrução educacional que valorizam o uso de estratégias metacognitivas na aprendizagem (Jou \& Sperb, 2006).

Uma das grandes mudanças no desenvolvimento, durante os anos escolares e na adolescência, é aprender a maximizar o uso das capacidades cognitivas através das capacidades metacognitivas (Miller, 1993). Blakey e Spence (2000) especificam mais as atividades metacognitivas, indicando três estratégias metacognitivas básicas: saber relacionar novas informações às já existentes; saber selecionar estratégias de pensamento propositadamente; saber planejar, monitorar e avaliar os processos de pensamento. Sob essa perspectiva, alunos que soubes- 
sem utilizar com eficiência as habilidades metacognitivas seriam aprendizes eficientes.

É importante destacar o fato de os pesquisadores dessa área terem estudado e apresentado, de forma explícita e sistemática, os mecanismos cognitivos envolvidos em um melhor desempenho da aprendizagem, permitindo que esses mecanismos sejam objeto de estudo e de análise. Esse conhecimento, portanto, permitiria aos professores trabalhar com o desenvolvimento das habilidades de aprendizagem em uma proposta de "aprender a aprender". Além disso, os profissionais que trabalham com indivíduos que apresentam dificuldade de aprendizagem podem desenvolver as habilidades específicas da aprendizagem, enfatizando os processos metacognitivos.

Vários trabalhos empíricos têm mostrado a metacognição como um fator determinante na aprendizagem formal. Por exemplo, Thiede e Anderson (2003) examinaram a relação entre a estratégia metacognitiva e a eficiência na compreensão de leitura, e observaram um efeito significativo do ensino dessas estratégias no desempenho dos alunos em compreensão de leitura. Os autores apontam para a importância da aprendizagem autorregulada no ensino formal. Schraw (1994), por sua vez, verificou que alunos universitários com habilidades precárias de monitoramento eram menos hábeis em gerenciar sua aprendizagem e tinham um desempenho inferior àqueles que eram bons monitores.

Desoete, Roeyers e De Clercq (2003) realizaram uma curta intervenção com instruções metacognitivas combinadas com instruções cognitivas algorítmicas para resolução de problemas. Participaram desse estudo 237 crianças da $3^{\text {a }}$ série do ensino fundamental. Os resultados mostraram que as crianças submetidas ao treinamento das habilidades metacognitivas tiveram melhor desempenho do que as crianças treinadas apenas nas habilidades cognitivas específicas para resolução de problemas.

Zohar e David (2008) também realizaram um estudo de intervenção, investigando a aprendizagem de biologia. Os pesquisadores, primeiramente, agruparam os alunos segundo o desempenho (médio e inferior). Esses dois grupos, por sua vez, foram divididos em grupo experimental, que recebeu instruções explícitas sobre o uso de estratégias metacognitivas, e grupo controle, que recebeu conhecimento específico de biologia. Os resultados indicaram que ensinar explicitamente metacognição teve um forte efeito nos alunos com desempenho mais baixo.

Sternberg e Spear-Swerling (1999) apontam para a importância de os professores entenderem o funcionamento metacognitivo de seus alunos e a sua interação com outros aspectos, como habilidades e fraquezas, personalidade e estilo de aprendizagem, para então empregarem metodologias mais adequadas, que proporcionem uma aprendizagem mais efetiva. O estudo de Erktin (2004) analisou o papel da metacognição no desempenho mate- mático e o efeito positivo das habilidades metacognitivas do próprio professor no desempenho dos alunos, introduzindo, no campo da educação formal, o debate sobre a possibilidade de ensinar estratégias de pensamento.

Jou e Sperb (2009) realizaram um estudo sobre leitura compreensiva, com duas turmas de alunos de $5^{\mathrm{a}}$ série, sendo uma o grupo experimental e a outra o grupo controle. A professora do grupo experimental foi capacitada a desenvolver as atividades de leitura, explicitando as estratégias metacognitivas específicas de leitura. Foram realizadas 16 sessões com leituras do livro-texto. O grupo controle leu os mesmos textos com sua professora, que não recebeu a capacitação. Os resultados mostraram que o grupo experimental aumentou significativamente suas habilidades de compreensão da leitura.

Os trabalhos empíricos desenvolvidos nas últimas décadas têm mostrado que a capacidade de monitorar e autorregular a própria cognição é um fator importante na aprendizagem, possibilitando a construção de um campo científico explícito e sistemático em relação aos mecanismos cognitivos e metacognitivos envolvidos no desempenho dos alunos. Isso tem permitido desenvolver um conhecimento sistemático acerca dos processos cognitivos da aprendizagem a serem utilizados na área educacional. A partir desses pressupostos, professores e psicólogos cognitivos poderiam desenvolver métodos de aprendizagem que propiciassem o surgimento e o progresso de capacidades cognitivas necessárias para amalgamar a grande quantidade de informações disponíveis no mundo (Jou \& Sperb, 2006).

Pergunta-se se o professor pode ser um mediador importante, ensinando habilidades cognitivas e metacognitivas, depois de ser treinado para isso? O objetivo principal desse estudo é relatar e avaliar um curso de capacitação de professores, baseado no conhecimento de tais habilidades aplicado à aprendizagem. A avaliação do efeito do curso de capacitação junto a um grupo de professores será feita através da avaliação do desempenho de seus alunos, comparado a um grupo controle (impacto da capacitação dos professores sobre os alunos) e, também, através da avaliação dos professores (impacto da capacitação sobre os próprios professores).

\section{Método}

\section{Delineamento}

Trata-se de um estudo de intervenção junto a professores com pré e pós-teste em seus alunos. As variáveis dependentes do estudo foram estratégias de aprendizagem, raciocínio, motivação, desempenho escolar e compreensão leitora nos alunos, e as variáveis independentes foram tempo e grupo.

\section{Participantes}

Participaram da pesquisa 54 alunos de $5^{\text {a }}$ série do ensino fundamental (32 alunos do grupo experimental e 22 
alunos do grupo controle), com idade média de 11 anos. Também participaram 10 professores dos 32 alunos do grupo experimental que realizaram o curso de capacitação.

\section{Instrumentos}

Foram utilizados para a coleta dos dados os seguintes instrumentos:

Alunos.

Fichas com os dados pessoais dos participantes.

Teste de Matrizes Progressivas Coloridas de Raven com a finalidade de avaliar o raciocínio dos alunos participantes.

Escala de Avaliação para Motivação para Aprender de Alunos do Ensino Fundamental (EMA; Neves \& Boruchovitch, 2004) - consta de 34 itens sobre motivação extrínseca e intrínseca com possibilidades de respostas: sempre, às vezes e nunca.

Escala de Estratégias de Aprendizagem (Boruchovitch et al., 2006) - consta de 20 perguntas sobre estratégias de aprendizagem e hábitos de estudo, em que o participante responde sim ou não.

Teste de Desempenho Escolar (TDE; Stein, 1994) - trata-se de uma avaliação de habilidades básicas de leitura, escrita e aritmética, obtendo um escore geral do desempenho escolar.

Tarefa para avaliar compreensão de leitura - mediante a leitura do texto: A Velha Contrabandista, extraído de um livro de leitura de $5^{\mathrm{a}}$ série, seguida de um questionário de múltipla escolha.

Professores

Fichas com os dados pessoais dos participantes.

Inventário Metacognitivo (Metacognitive Awareness Inventory [MAI]; Schraw \& Dennison, 1994) - para os professores. Consta de 53 perguntas com respostas de falso ou verdadeiro.

Questionário de auto-avaliação para os professores - sobre o impacto do curso na sua atuação em sala de aula.

\section{Procedimentos}

Foram contatadas duas escolas estaduais que aceitaram participar da pesquisa. A escola A, por já ter realizado outros trabalhos com as pesquisadoras e devido ao interesse manifestado em relação a algum tipo de intervenção com os professores, foi escolhida como grupo experimental. A escola B foi convidada a participar como grupo controle por abranger um público com nível socioeconômico semelhante e por estar localizada em um bairro próximo da escola $\mathrm{A}$. Na escola A, portanto, realizou-se o trabalho de capacitação com os professores das $5^{\text {as }}$ séries e a aplicação dos instrumentos no pré e no pós-teste com os alunos das mesmas turmas. A escola disponibilizou os horários e o espaço para realização da capacitação. Na escola B (grupo controle) foi realizado pré e pós-teste nos alunos de uma turma de $5^{\text {a }}$ sé- rie. As duas escolas manifestaram interesse em participar da pesquisa.

A direção das escolas, os professores e os responsáveis por cada aluno foram esclarecidos quanto aos objetivos da pesquisa e, aqueles que aceitaram, assinaram o Termo de Consentimento Livre e Esclarecido. Os instrumentos foram aplicados de forma coletiva, sendo utilizados dois períodos de 45 minutos em dois dias consecutivos. A ordem de aplicação obedeceu ao critério de não sobrecarregar a atividade dos alunos. No primeiro dia foi aplicado o Raven e o texto de compreensão e no dia seguinte as tarefas do TDE e as escalas. Os professores responderam aos instrumentos no primeiro e no último dia do curso.

O pré-teste foi realizado no mês de abril de 2008 e o pós-teste no mês de dezembro do mesmo ano. O curso de capacitação ocorreu entre abril e setembro de 2008, uma vez por semana, totalizando 60 horas/aula. A capacitação teve formato de Curso de Extensão do Programa de PósGraduação em Psicologia da Universidade Federal do Rio Grande do Sul. Ao término do curso, os professores receberam um certificado de participação.

As aulas foram ministradas na própria escola, pelas pesquisadoras. O foco do curso foi o estudo do funcionamento do sistema cognitivo, desdobrado nos seguintes temas: metacognição e seu desenvolvimento; inteligência e racionalidade; memória e aprendizado; atenção seletiva; representação mental; leitura compreensiva; emoção e motivação; neuropsicologia da aprendizagem; dificuldades de aprendizagem. A dinâmica dos encontros oportunizou a leitura de artigos científicos, a exposição dos conceitos e discussão de casos expostos pelos professores. Também foi enfatizado o planejamento de atividades a serem realizadas com os alunos, com a finalidade de propiciar o desenvolvimento de estratégias cognitivas e metacognitivas de aprendizagem, com posterior avaliação das mesmas.

\section{Análise dos Dados}

Foi utilizada a ANOVA para comparação de grupos.

\section{Resultados e Discussão}

Com a finalidade de obter uma melhor compreensão dos dados, os resultados serão descritos através da comparação entre o pré e pós-teste de todos os instrumentos utilizados.

A análise do nível de desempenho escolar dos alunos foi realizada a partir da aplicação do Teste de Desempenho Escolar, que avalia os conhecimentos aritméticos e de escrita, indicando se o escore dos indivíduos é inferior, médio ou superior em relação a sua idade e escolaridade. Assim, os resultados indicaram que, em aritmética, $72 \%$ dos alunos do grupo experimental tiveram desempenho inferior no pré-teste ao passo que, no pósteste, $44 \%$ dos alunos ainda permaneceram nesse nível. 
Busnello, F. B., Jou, G. I. \& Sperb, T. M. (2012). Desenvolvimento de Habilidades Metacognitivas: Capacitação de Professores de Ensino Fundamental.

No pré-teste do grupo controle, $50 \%$ dos alunos estavam no nível inferior e, no pós-teste, $41 \%$ se mantiveram inferiores. Quanto ao desempenho na escrita, os resultados foram semelhantes aos de aritmética. No grupo experimental, $75 \%$ dos alunos no pré-teste e $47 \%$ no pós-teste tiveram desempenho inferior, enquanto que, no grupo controle, $68 \%$ no pré-teste e $50 \%$ no pós-teste tiveram desempenho inferior. Esses resultados vão ao encontro de avaliações nacionais e internacionais, que apontam para o baixo desempenho dos alunos de ensino fundamental no nosso país.

No ano de 2007, o desempenho dos alunos brasileiros ficou em $48^{\circ}$ lugar em leitura e em $53^{\circ}$ lugar em matemática, entre os 56 países participantes do Programa Internacional de Avaliação de Alunos (PISA). Os resultados do exame nacional do Sistema de Avaliação da Educação Básica (SAEB) de 2007 também indicam o baixo desempenho dos estudantes brasileiros nas provas de matemática e português, ainda que apontem para uma melhora no desempenho dos alunos de 2005 para 2007 nas séries avaliadas (Góis \& Pinho, 2007).

Com relação às variáveis estudadas, a ANOVA com medidas repetidas constatou que houve diferença significativa entre o pré e o pós-teste no Teste de Desempenho Escolar (aritmética e escrita), no Teste de Matrizes Progressivas e na Escala de motivação para aprendizagem, apontando para o efeito principal do tempo. A Tabela 1 expõe as médias e desvios padrões das variáveis no pré e pós-teste dos grupos experimental e con- trole e do total de alunos. A Tabela 2 mostra a comparação de médias e desvios padrões das variáveis no pré e pós-teste dos grupos experimental e controle. Esses resultados constataram o efeito tempo, nas variáveis mencionadas, entretanto o efeito grupo, isto é, o efeito da capacitação dos professores no desempenho dos alunos, foi percebido apenas para a variável estratégias de aprendizagem, indicando o efeito da interação grupo/ tempo (ver Tabela 2).

Especificamente, nas variáveis de desempenho (aritmética e escrita), houve diferença significativa por tempo, mas não foram observadas diferenças significativas entre o grupo experimental e o grupo controle. Isso significa que os alunos obtiveram a melhora esperada na aprendizagem em decorrência do próprio ano letivo, mas não apareceu o efeito grupo, relacionado às possíveis mudanças pedagógicas dos professores.

Com relação à leitura compreensiva, não houve diferença significativa para o efeito tempo e grupo, isto é, os alunos nos dois grupos não melhoraram sua capacidade de leitura ao final do ano letivo. Cabe destacar que o desempenho alto, nos pré e pós-teste no questionário de múltipla escolha, pode apontar para um efeito de teto (ceiling effect) na tarefa de compreensão. A esse respeito, salienta-se que, embora os testes de múltipla escolha sejam aceitos como medidas de memória para avaliar compreensão de texto (Vega, Carreiras, Gutiérrez-Calvo, \& Alonso-Quecuty, 1990), os resultados neste trabalho levam a questionar a adequação do teste utilizado.

Tabela 1

Médias e Desvios Padrões das Variáveis no Pré e Pós-teste dos Grupos Experimental e Controle e do Total de Alunos

\begin{tabular}{lcrrrr}
\hline \multirow{2}{*}{ Variaveis } & Tempo & \multicolumn{3}{c}{ Grupo } \\
\cline { 3 - 6 } & \multicolumn{2}{c}{ Experimental } & \multicolumn{2}{c}{ Controle } \\
& & Média & $D P$ & Média & $D P$ \\
\hline \multirow{2}{*}{ RAVEN } & Pré & 29,81 & 4,17 & 29,32 & 5,06 \\
& Pós & 31,53 & 3,89 & 31,23 & 3,37 \\
TDEA & Pré & 19,53 & 2,7 & 21,14 & 4,43 \\
& Pós & 21,66 & 3,52 & 22,42 & 4,14 \\
TDEE & Pré & 26,13 & 3,85 & 25,86 & 5,17 \\
& Pós & 27,72 & 3,72 & 28,32 & 3,67 \\
Comp. Leitora & Pré & 8,38 & 1,36 & 8,41 & 1,68 \\
& Pós & 8,41 & 1,1 & 8,36 & 1,18 \\
EEA & Pré & 8,44 & 3,11 & 9 & 3,35 \\
\multirow{2}{*}{ EMA } & Pós & 9,41 & 2,83 & 8,09 & 3,4 \\
& Pré & 88 & 9,7 & 76,32 & 11,88 \\
& Pós & 81,41 & 11,09 & 74,59 & 10,97 \\
\hline
\end{tabular}

Notas . Raven $=$ Teste de Matrizes Progressivas de Raven; TDEA = Teste de desempenho escolar de aritmética; TDEA $=$ Teste de desempenho escolar de escrita; Comp.Leitura = Compreensão Leitora; EEA = Escala de Estratégias de Aprendizagem; EMA = Escala de Motivação para a Aprendizagem. 
Tabela 2

Comparação de Médias e Desvios Padrões das Variáveis no Pré e Pós-teste dos Grupos Experimental e Controle (ANOVA, medidas repetidas)

\begin{tabular}{lllll}
\hline Variáveis & Causas da variância & $g l$ & $F$ & $P$ \\
\hline \multirow{2}{*}{ RAVEN } & tempo & 1 & 22,18 & $0,001^{*}$ \\
& grupo & 1 & 0,136 & 0,713 \\
& tempo*grupo & 1 & 0,061 & 0,806 \\
TDEA & tempo & 1 & 11,1 & $0,002^{*}$ \\
& grupo & 1 & 1,92 & 0,172 \\
& tempo*grupo & 1 & 0,61 & 0,439 \\
TDEE & tempo & 1 & 14,95 & $0,000^{*}$ \\
& grupo & 1 & 1,80 & 0,186 \\
& tempo*grupo & 1 & 0,68 & 0,415 \\
Comp. Leitora & tempo & 1 & 0,002 & 0,97 \\
& grupo & 1 & 0,000 & 0,989 \\
& tempo*grupo & 1 & 0,044 & 0,834 \\
EEA & tempo & 1 & 0,005 & 0,0943 \\
& grupo & 1 & 0,258 & 0,614 \\
& tempo*grupo & 1 & 5,17 & $0,027^{*}$ \\
EMA & tempo & 1 & 8,96 & $0,004^{*}$ \\
& grupo & 1 & 12,09 & $0,001^{*}$ \\
\hline
\end{tabular}

Notas . Raven $=$ Teste de Matrizes Progressivas de Raven; TDEA = Teste de desempenho escolar de aritmética; TDEA = Teste de desempenho escolar de escrita; Comp.Leitura = Compreensão Leitora; EEA = Escala de Estratégias de Aprendizagem; EMA = Escala de Motivação para a Aprendizagem.

$* p<0,05$.

Os testes de múltipla escolha são mais direcionados aos processos de reconhecimento na evocação dos conteúdos da memória, dependendo mais de processos associativos da memória semântica e da informação armazenada na memória de curto prazo (MCP). Após a leitura, as perguntas específicas sobre dados do texto permitiriam evocar rapidamente o conhecimento recém armazenado. Dessa forma, esse processo de evocação não exige uma busca seletiva de informação armazenada, como também não exige uma organização desses dados. Entretanto, o processo de recordação livre exige do sujeito uma busca seletiva das informações que corresponde à organização que nossa mente deu a esses conhecimentos. Em geral, essa organização respeita a coerência e a compreensão do texto recuperado. Neste caso, poder-se-ia pensar que o questionário de múltipla escolha estaria medindo apenas a ativação de informações estocadas na MCP e não a compreensão mais profunda do texto, facilitando a tarefa realizada.

Com relação às estratégias de aprendizagem, observou-se uma interação significativa de tempo e grupo. Enquanto o grupo experimental mostrou um aumento das estratégias de aprendizagem apontadas na escala, observou-se que o grupo controle diminuiu a utilização de tais estra-tégias. Esse resultado sugere um possível efeito do trabalho realizado pelos professores, incentivando o uso de estratégias específicas para aprender (ver Tabela 3).

Tabela 3

Comparação de Médias e Desvios Padrões da Variável: Estratégias de Aprendizagem no Pré e Pós-teste dos Grupos Experimental e Controle (ANOVA, medidas repetidas)

\begin{tabular}{|c|c|c|c|c|c|}
\hline \multirow{3}{*}{$\begin{array}{l}\text { Estratégias de } \\
\text { Aprendizagem }\end{array}$} & & \multicolumn{4}{|c|}{ Grupos } \\
\hline & & \multicolumn{2}{|c|}{$\begin{array}{c}\text { Experimental } \\
\quad n=32\end{array}$} & \multicolumn{2}{|c|}{$\begin{array}{c}\text { Controle } \\
n=22\end{array}$} \\
\hline & & Média & $D P$ & Média & $D P$ \\
\hline & Pré & $8,44^{\mathrm{AB}}$ & 3,11 & $9,00^{\mathrm{AB}}$ & 3,35 \\
\hline & Pós & $9,41^{\mathrm{A}}$ & 2,83 & $8,09^{\mathrm{B}}$ & 3,04 \\
\hline
\end{tabular}

Nota. Médias seguidas de letras distintas diferem significativamente através da Análise de variância, utilizando o delineamento de medidas repetidas, complementada pelo teste de comparações Múltiples de Tukey, ao nível de significância de 5\%.

Outro aspecto importante a ser destacado nesta análise é o decréscimo no nível de motivação para aprendizagem apresentado pelos alunos de ambos os grupos entre o pré 
e o pós-teste. Esse fato pode ser justificado pela atitude da maioria dos alunos no mês de dezembro, período em que foi realizado o pós-teste. Uma vez terminadas as provas finais, as crianças não estariam tão motivadas para aprender como no começo do ano.

Também cabe mencionar o percentil acima da média que a maioria dos alunos obteve no desempenho do teste de Matrizes Progressivas, tanto no pré-teste quanto no pós-teste. De acordo com a análise dos resultados do Teste de Matrizes Progressivas de Raven, observou-se que a grande maioria dos alunos apresenta inteligência média ou superior, mostrando uma boa capacidade de raciocínio. Tal desempenho mostra uma discrepância com o baixo desempenho dos alunos nas tarefas do conhecimento, já que um índice expressivo de alunos obteve desempenho inferior nas tarefas referentes à aritmética e escrita.

Com a finalidade de entender a divergência entre nível intelectual e desempenho, analisou-se o tipo de erro, verificando-se que o desempenho na aritmética foi prejudicado por erros ligados à atenção. Ao realizar um cálculo, por exemplo, a criança utilizava o procedimento de "levar um", mas não o somava no resultado final. Erros de perseverança também apontaram mais para a falta de atenção do que para a falta de aprendizagem da aritmética. Nesses casos, a criança, após uma sequência de cálculos de soma, não percebia o início de uma nova sequência de cálculos de subtração, e continuava somando. Da mesma forma, os erros de escrita consistiam em não colocar uma letra ou na dificuldade de reter a informação quando a palavra era composta de mais de três sílabas. Frente a essas constatações, pode-se concluir que o baixo desempenho dos alunos estudados corresponde mais às falhas nas habilidades cognitivas e metacognitivas do que ao baixo potencial intelectual ou à dificuldade de aprendizagem do conhecimento específico.

Esse dado aponta, por um lado, para a necessidade de que as habilidades cognitivas e metacognitivas para aprendizagem sejam desenvolvidas nos anos escolares, como sugere Resnick (1981). Por outro lado, ressalta-se a dificuldade de que tais habilidades sejam desenvolvidas em sala de aula, já que os resultados mostraram que apenas as estratégias de aprendizagem tiveram diferença significativa entre o pré e o pós-teste do grupo experimental. Contudo cabe supor que, se os alunos incorporaram, de forma explícita, mais estratégias de aprendizagem, possivelmente consigam manifestá-las, posteriormente, no seu desempenho escolar. Imagina-se, assim, que a manifestação das estratégias de aprendizagem no desempenho dos estudantes possa ocorrer a partir da mediação dos professores, exercitando de forma contínua essas estratégias com seus alunos, possibilitando a generalização das habilidades cognitivas e metacognitivas da aprendizagem para outras atividades do seu cotidiano.

Com relação à avaliação dos professores, o inventário metacognitivo permitiu constatar que os professores participantes tiveram um bom desempenho, atingindo uma porcentagem média de $78 \%$ na escala. Por outro lado, com a finalidade de avaliar o processo de capacitação, os professores responderam um questionário de autoavaliação e no último encontro, o grupo avaliou o impacto do curso na atuação em sala de aula. Os professores manifestaram, de forma geral, terem introduzido estratégias para desenvolver as capacidades metacognitivas dos alunos, como refletir sobre o processo de aprendizagem, planejar a realização das tarefas escolares, controlarem a atenção, entre outras. Também foi relatado que as mudanças na metodologia pedagógica dos professores foram percebidas pelos alunos, segundo foi reportado pelos próprios professores.

Os professores tiveram a oportunidade de aplicar, nas suas aulas, as diferentes estratégias de ensino/aprendizagem planejadas nos encontros para, posteriormente, discutirem-nas no grupo. Deve-se salientar o interesse e a motivação dos professores ao longo do curso, ratificados pela presença assídua e participação constante.

Com relação à motivação, uma professora declarou que a apresentação de objetivos claros e estratégias planejadas tinha-lhe trazido a motivação de volta, pois se sentia pouco motivada como professora. Essa colocação foi discutida no grupo, criando um desafio para pesquisar sobre motivação humana. A pesquisadora procurou um referencial teórico que focasse especificamente a relação entre objetivos/estratégias e motivação, e encontrou artigos referentes à teoria de motivação humana por fixação de objetivos, de Edwin Locke e Gary Latham (2002). Como esta abordagem não estava incluída no capítulo de motivação do curso de capacitação, abriu-se um espaço para discussão sobre essa teoria, o que permitiu aos professores se manifestarem sobre suas variáveis motivacionais.

Todos apontaram que utilizar rotinas sistemáticas, como estimular mais a reflexão sobre o que tinham apreendido e sobre como tinham aprendido antes de finalizar a aula, deu-lhes a oportunidade de repensar suas estratégias de ensino. Os professores também indicaram uma maior utilização de diagramas e esquemas, trabalhando mais a capacidade de sínteses de seus alunos. Observou-se que muitas das propostas sugeridas no curso já eram utilizadas esporadicamente pelos professores que, ao conhecerem o embasamento teórico subjacente às estratégias sugeridas, sentiram-se mais motivados a utilizar essas rotinas de forma sistemática.

A esse respeito, uma professora disse: "Eu já fiz isso, mas não tinha certeza se ajudava na aprendizagem". Esse comentário foi discutido, levantando-se a importância de eles mesmos poderem analisar o efeito de suas inovações.

A própria avaliação dos professores, solicitada de forma escrita, mostra as mudanças provocadas pelo curso. A seguir serão transcritos trechos das avaliações dos professores.

"O curso foi bem específico, com resultados imediatos, pois já podíamos aplicar em sala de aula, podendo sempre ter um feedback, consultando com a professora ou mesmo no polígrafo". 
"Trabalhamos conceitos sobre a cognição de forma teórica e aplicamos simultaneamente no contexto da sala de aula, de forma a desenvolver as capacidades cognitivas e metacognitivas".

"Todos os processos e comportamentos que, durante o curso, definimos e caracterizamos, como atenção, memória, percepção, emoções, motivação e auto-estima, foram muito esclarecedores e envolventes. Serviram, posteriormente, como ferramentas para aplicarmos no trabalho diário em sala de aula".

"O referente curso predispõe ao educador a uma diversidade de alternativas de organização de ações, desencadeando um resultado mais efetivo na aprendizagem, como também, proporcionando ao educando uma maior motivação, levando-o a se sentir um aluno ativo, importante no processo".

"O curso foi uma excelente oportunidade que tivemos para nos reciclar. A universidade veio até nós, teve esse mérito e nos envolveu no aprofundamento e maior conhecimento para podermos conhecer melhor os processos que envolvem a alma do estudante. Essa importante ferramenta nos permitiu a troca de experiências e aplicação de processos. Efeitos imediatos já se fazem presentes nas experiências que vivenciamos e, tenho certeza, outros frutos virão por conta dessas experiências".

"O que pude perceber é que a sistematização dos conteúdos aplicados acaba fazendo a diferença nos resultados. Muitas vezes, temos consciência dos métodos, já os utilizamos, porém, não de forma sistemática, que é o que realmente produz resultados. Porém, agora é importante darmos continuidade ao trabalho desenvolvido, para termos melhores resultados a médio e longo prazo".

\section{Considerações Finais}

Os resultados mostram que o curso de capacitação teve um impacto importante, provocando mudanças nas ações pedagógicas e na motivação dos professores participantes. Embora essas mudanças não tenham aparecido no desempenho em aritmética, escrita e compreensão leitora, elas foram percebidas a partir do aumento do uso de estratégias de aprendizagem apontadas pelos alunos no pós-teste, podendo ser considerado um primeiro passo em direção à melhora no desempenho escolar. Esse fato permite supor que o professor pode ser um multiplicador e mediador de mudanças cognitivas e metacognitivas nos alunos, de acordo com a proposta de Resnick (1981), ao afirmar que as práticas educacionais baseadas na psicologia cognitiva podem desenvolver as habilidades cognitivas, metacognitivas e motivacionais dos alunos no ensino formal.

Considerando que mudanças demoram a acontecer, é possível pensar que, prolongando a duração da intervenção, as crianças poderiam incorporar, em seu cotidiano, as estratégias cognitivas e metacognitivas trabalhadas pelos professores, de forma que essas mudanças fossem observadas diretamente no desempenho escolar.
Os alunos muito se beneficiariam, especificamente, com o desenvolvimento de estratégias metacognitivas que levam a criança a refletir e revisar suas tarefas. Dessa forma, os erros cometidos pelos alunos nas tarefas de aritmética e escrita seriam minimizados se a criança tivesse como rotina revisar e corrigir as atividades antes de entregar, ou monitorando os procedimentos enquanto os está realizando.

Os resultados encontrados evidenciam a necessidade de outros estudos sobre a qualificação de professores do ensino fundamental, especialmente quanto ao desenvolvimento das habilidades cognitivas e metacognitivas na aprendizagem dos estudantes. Ademais, os achados desta pesquisa sugerem que o curso de capacitação realizado com os professores surtiria mais efeito no desempenho acadêmico dos estudantes se contemplasse um período maior de duração, permitindo enfatizar as práticas em sala de aula e a implementação de novas estratégias cognitivas e metacognitivas na rotina dos alunos, transformando uma atividade eventual em atividade sistemática. Assim, espera-se que a presente pesquisa possa contribuir de alguma maneira para a busca de um melhor desempenho de nossos alunos para que, num futuro não muito distante, esses estudantes possam figurar nos primeiros lugares das avaliações educacionais.

\section{Referências}

Armbruster, B. B., \& Brown, A. L. (1984). Learning from reading: The role of meta-cognition. In R. C. Anderson, J. Osborn, \& R. J. Tierney (Eds.), Learning to read in American schools: Basal readers and content texts. Hillsdale, NJ: Lawrence Erlbaum.

Bieleaczyc, K., Pirolli, P. L., \& Brown, A. L (1995). Training in self-explanation and self-regulation strategies: Investigating the effect of knowledge acquisition activities on problem solving. Cognition and Instruction, 13(2), 221-252.

Blakey, E., \& Spence, S. (2000). Developing metacognition. Retrieved June 8, 2009, from http://www.ericdigests.org/pre9218/developing.htm

Boruchovitch, E., \& Mercuri, E. (1999). A importância do sublinhar como estratégia de estudo de textos. Revista Tecnologia Educacional, 28, 37-40.

Boruchovitch, E., Santos, A. A. A., Costa, E. R., Neves, E. R. C., Cruvinel, M., Primi, R., et al. (2006). A construção de uma escala de estratégias de aprendizagem para alunos do ensino fundamental. Psicologia: Teoria e Pesquisa, 22, 297-304.

Bosa, C. A. (2001). As relações entre autismo, comportamento social e função executiva. Psicologia: Reflexão e Critica, 14(2). Retrieved from http://www.scielo.br/scielo

Davis, C., Nunes, M. M. R., \& Nunes, C. A. A. (2005). Metacognição e sucesso escolar: Articulando teoria e prática. Cadernos de Pesquisa, 35(125), 205-230.

Desoete, A., Roeyers, H., \& De Clercq, A. (2003). Can offline metacognition enhance mathematical problem solving? Journal of Educational Psychology, 95(1), 188-200.

Erktin, E. (2004). Teaching thinking for mathematics through the enhancement of metacognitives skills. Research in the Schools, 11(1), 3-13. 
Busnello, F. B., Jou, G. I. \& Sperb, T. M. (2012). Desenvolvimento de Habilidades Metacognitivas: Capacitação de Professores de Ensino Fundamental.

Ertmer, P. A., \& Newby, T. J. (1996). The expert learner: Strategies, self-regulated, and reflective. Instructional Science 24, 1-24.

Flavell, J. (1987). Speculations about the nature and development of metacognition. In F. Weinert \& R. Kluwe (Eds.), Metacognition, motivation, and understanding (pp. 21-29). Hillsdale, NJ: Lawrence Erlbaum.

Forest-Pressley, D. L., \& Waller, T. G. (1984). Cognition, metacognition and reading. New York: Springer-Verlag.

Garner, R. (1987). Metacognition and reading comprehension. Norwood, NJ: Ablex.

Góis, A., \& Pinho, A. (2007). Brasil é reprovado, de novo, em matemática e leitura. Folha.com. Retrieved from http:// www 1 . fol ha.uol.com.br/fol ha/educacao/ ult305u351481.shtml

Hartman, H. J. (2002). Teaching metacognitively. In H. J. Hartman (Ed.), Metacognition in learning and instruction (pp. 149-164). Boston: Kluwer Academic.

Jou, G. I. (2001). As habilidades cognitivas na compreensão da leitura: Um processo de intervenção no contexto escolar. (Tese de Doutorado não-publicada). Universidade Federal do Rio Grande do Sul, Porto Alegre, RS.

Jou, G. I., \& Sperb, T. M. (2006). A metacognição como estratégia reguladora da aparendizagem. Psicologia: Reflexão e Crítica, 19, 12-20.

Jou, G. I., \& Sperb, T. M. (2008). Leitura compreensiva: O processo instrucional. Linguagem \& Ensino (Pelotas), 11, 145-177.

Jou, G. I., \& Sperb, T. M. (2009). Lectura comprensiva: estudo de intervención. Interamerican Journal of Psychology, 43, 12-21.

Klauer, K. J. (1994). Cognitive training for children. A developmental program of inductive reasoning and problem solving. Seattle, WA: Hogrefe \& Huber.

Kramarski, B., \& Michalsky, T. (2009). Investigating preservice teachers' professional growth in self-regulated learning environments. Journal of Educational Psychology, 101(1), 161-175.

Locke, E. A., \& Latham, G. P. (2002). New directions in goalsetting theory. Current Directions in Psychological Science, 15(5), 265-268.

Miller, P. H. (1993). Theories of Developmental Psychology. New York: W.H. Freeman.

Nelson, T., \& Narens, L. (1996). Why investigate metacognition? In J. Metcalfe \& A. P. Shimamura (Eds.), Metacognition. Knowing about knowing (pp. 1-27). Cambridge, MA: Massachusetts Institute of Technology Press.

Neves, E. R. C., \& Boruchovitch, E. (2004). A motivação de alunos no contexto da progressão continuada. Psicologia: Teoria e Pesquisa, 20(1), 77-85.

Oliveira, A., Lima, B., Roberty, B., Rodrigues, D. M., Aires, J., Chahon, M., et al. (1989). The challenges of classroom strategy instruction. The Elementary School Journal, 89, 301342.

Resnick, L. B. (1981). Instructional Psychology. Annual Review of Psychology, 32, 659-704.

Rivers, W. P. (2001). Autonomy at all costs: An ethnography of metacognitive self-assessment and self- management among experienced language learners. The Modern Language Journal, 85(2), 279-290.
Schaefer, L., Pavan, C., Amaral, B., \& Jou, G. I. (2006). Desenvolvimento da habilidade de aprendizagem: Um estudo com aprendizes eficientes. Trabalho apresentado no XVIII Salão de Iniciação Científica, Universidade Federal do Rio Grande do Sul, Porto Alegre, RS.

Salas, E., \& Cannon-Bowers, J. (2001). The science of training: A decade of progress. Annual Review of Psychology, 52, 471499.

Schraw, G. (1994). The effect of metacognitive knowledge on local and global monitoring. Contemporary Educational Psychology, 19, 143-154.

Schraw, G., Crippen, K. J., \& Hartley, K. D. (2006). Promoting self-regulation in science education: Metacognition as part of a broader perspective on learning. Research in Science Education, 36(1/2), 111-139.

Schraw, G., \& Dennidon, R. S. (1994). Assessing metacognitive awareness. Contemporary Educational Psychology, 19(4), 460-475.

Stein, L. M. (1994). TDE - Teste de Desempenho Escolar: Manual para aplicação e interpretação. São Paulo, SP: Casa do Psicólogo.

Sternberg, R. J. (2002). Metacognition, abilities, and developing expertise: What makes an expert student? In H. J. Hartman (Ed.), Metacognition in learning and instruction (pp. 149164). Boston: Kluwer Academic.

Sternberg, R. J., \& Spear-Swerling, L. (1999). Enseñar a pensar. Madrid, España: Grupo Santillana de Ediciones.

Thiede, K. W., \& Anderson, M. C. M. (2003). Summarizing can improve metacomprehension accuracy. Contemporary Educational Psychology, 28, 129-160.

Vega, M., Gutiérrez-Calvo, M., \& Alonso-Quecuty, M. L. (1990). Lectura y comprensión: uma perspectiva cognitiva. Madrid, España: Alianza.

Vidal-Abarca, E. (1990). Um programa para la enseñanza de la comprensión de ideas principales de textos expositivos. Infancia y Aprendizaje, 49, 53-71.

Vieira, E. (1999). Intervenção psicopedagógica na fase de representação mental em resolução de problemas matemáticos. (Tese de Doutorado não-publicada). Pontifícia Universidade Católica do Rio Grande do Sul, Porto Alegre, RS.

Zimmerman, B. J. (2001). Theories of self-regulated learning and academic achievement: An overview and analysis. In B. J. Zimmerman \& D. H. Schunk (Eds.), Self-regulated learning and academic achievement: Theoretical perspectives (pp. 138). Mahwah, NJ: Lawrence Erlbaum.

Zohar, A., \& David, A. B. (2008). Explicit teaching of metastrategic knowledge in authentic classroom situations. Metacognition Learning, 3, 59-82. 